\title{
Formação de Grupos em Ambientes CSCL baseada na combinação entre os Traços de Personalidade e Teorias de Aprendizagem Colaborativa
}

\author{
Rachel C. D. Reis ${ }^{1,2}$, Seiji Isotani ${ }^{1}$ \\ ${ }^{1}$ Instituto de Ciências Matemáticas e de Computação - Universidade de São Paulo \\ Avenida Trabalhador São Carlense, 400 - Centro - 13566-590 - São Carlos - SP \\ ${ }^{2}$ Universidade Federal de Viçosa, Campus Rio Paranaíba, Rio Paranaíba - MG. \\ rachel.reis@ufv.br, sisotani@icmc.usp.br
}

\begin{abstract}
Group formation in CSCL environments is considered one of the essential elements for the success of collaborative learning. Despite of important contributions, we observed the lack of studies to match personality traits to collaborative learning theories. Therefore, this paper aims at verifying the influence of personality traits on group formation based on learning theories and creating mechanisms to automate such formation. As main results, we present the creation of a Formal Model for the generation of new learning roles (PCAs) and the development of an algorithm that uses these PCAs to support the formation of more effective groups.
\end{abstract}

Resumo. A formação de grupos em ambientes CSCL é considerada um dos elementos essenciais para o sucesso da aprendizagem colaborativa. Apesar das importantes contribuições, observa-se a carência de estudos que relacionem os traços de personalidade às teorias de aprendizagem colaborativa. Logo, este artigo tem como objetivo verificar a influência dos traços de personalidade na formação de grupos baseados em teorias de aprendizagem e criar mecanismos para automatizar essa formação. Como principais resultados tem-se a criação de um Modelo Formal para geração de novos papéis de aprendizagem (PCAs) e o desenvolvimento de um algoritmo que utilize esses PCAs para apoiar a formação de grupos mais efetivos.

\section{Introdução}

A formação de grupos em ambientes de Aprendizagem Colaborativa com Suporte Computacional (do inglês Computer Supported Collaborative Learning - CSCL) representa a estrutura básica para a criação de cenários de aprendizagem que promovam a colaboração entre duas ou mais pessoas [Isotani et al. 2009]. Pesquisadores na literatura ressaltam a preocupação sobre as formações baseadas em estratégias que podem resultar em participação desigual dos membros nos grupos [Isotani e Mizoguchi 2008], que podem ocorrer devido à falta de planejamento e seleção inadequada dos fatores e mecanismos de colaboração [Borges et al. 2018]. No intuito de mapear as soluções existentes, Borges et al. (2018) mostram que os fatores mais investigados na formação de grupos referem-se ao nível de conhecimento dos alunos, seguido das habilidades e papel dos aprendizes. 
Inaba e Mizoguchi (2004) enfatizam a importância de considerar a atribuição de papéis na formação de grupos em ambientes CSCL, uma vez que este elemento pode ser um valioso recurso para promover a consciência dos estudantes no processo de colaboração e interação com os colegas. No entanto, se o aprendiz desempenhar um papel que não seja adequado ao seu perfil, os benefícios educacionais podem não ser alcançados, e como consequência, toda a atividade colaborativa pode ser prejudicada. Para mitigar este problema, projetistas instrucionais têm usado as teorias de aprendizagem colaborativa como diretrizes para a projeção dos cenários de aprendizagem mais efetivos [Isotani et al. 2009]. Essas teorias explicam o processo de aprendizagem a partir da definição de fatores essenciais para o aprendizado em grupo. Por exemplo, o contexto para desenvolvimento das atividades em grupo, o tipo de conhecimento e habilidade a serem desenvolvidos, o papel a ser desempenhado pelos aprendizes, os padrões de interação recomendados para cada papel, dentre outros. Alguns exemplos de teorias de aprendizagem colaborativa são Anchored Instruction [CTGV 1992] e Peer Tutoring [Endlsey 1980].

Outro fator importante considerado na formação de grupos são os traços de personalidade [Reis et al. 2015]. Pesquisadores destacam a importância dos traços de personalidade como elementos críticos que afetam a colaboração e a interação dos estudantes no trabalho em grupo, dado que este fator pode influenciar o desempenho e satisfação dos estudantes e induzir diversas ações e comportamentos no trabalho em grupo [Reis et al. 2017, Andrei et al. 2015, Molleman et al. 2004, Peeters et al. 2006].

Com respeito ao uso dos traços de personalidade na formação de grupos em ambientes CSCL, Reis et al. (2015) ${ }^{1}$ constataram por meio de uma revisão da literatura que, em geral, esses elementos são usados sem a combinação com outros fatores importantes para a organização dos grupos, tais como: teorias de aprendizagem, papéis, padrões de interação, objetivos individuais, dentre outros [Isotani et al., 2009]. Além disso, as estratégias de agrupamento utilizadas para modelagem dos traços de personalidade em sistemas computacionais baseiam-se em diferentes técnicas como, algoritmos genéticos, algoritmos de agrupamento, modelos matemáticos, modelos de processo, entre outros [Reis et al. 2015]. Essas técnicas têm sido utilizadas, por exemplo, para apoiar a formação de grupos a partir da criação de (1) modelos de predição para prever o desempenho dos alunos na aprendizagem colaborativa [Farhangian et al. 2015]; (2) sistemas de recomendação para recomendar equipes de trabalho com base na similaridade dos traços de personalidade dos alunos e seus tutores [Nunes et al. 2010]; (3) modelos de processo para apoiar a formação de grupos em ambientes de aprendizagem aberto (MOOC) [Spoelstra et al. 2014], dentre outros.

Apesar das importantes contribuições dos trabalhos sobre formação de grupos em ambientes CSCL, observa-se que são poucos os trabalhos na literatura que combinam os traços de personalidade a outros fatores importantes no agrupamento dos alunos [Reis et al. 2015]. Além disso, são raras as pesquisas que se baseiam em abordagens pedagógicas, fundamentadas em teorias de aprendizagem, e que considerem os traços de personalidade para apoiar a formação de grupos [Borges et al. 2018].

\footnotetext{
${ }^{1}$ A revisão da literatura (mapeamento sistemático) desenvolvida por Reis et al. (2015) foi realizada pelos autores durante o desenvolvimento deste trabalho de doutorado.
} 
Logo, este trabalho tem como objetivo verificar a influência dos traços de personalidade na formação de grupos baseados em teorias de aprendizagem colaborativa e criar mecanismos para automatizar e apoiar essa formação. Para alcançar esse objetivo, este trabalho é norteado por três questões de pesquisas com o intuito de (1) investigar a relação entre os traços de personalidade e as variáveis de aprendizado, satisfação e motivação dos grupos baseados em teorias de aprendizagem colaborativa; (2) criar um modelo formal que relacione os traços de personalidade às teorias de aprendizagem colaborativa para a geração de novos papéis de aprendizagem; (3) desenvolver um algoritmo computacional que utilize os novos papéis e viabilize a formação de grupos de aprendizagem mais efetivos.

Além dessa seção introdutória que apresentou a área de formação de grupos em ambientes CSCL e pesquisas relacionadas, este trabalho está organizado da seguinte forma: a Seção 2 apresenta o método de pesquisa para o desenvolvimento deste trabalho. A Seção 3 descreve as questões de pesquisa que guiaram a realização desse trabalho e os resultados obtidos. Finalmente, na Seção 4 são apresentadas as conclusões.

\section{Método}

Seguindo a conceituação metodológica apresentada por Jung (2004), este trabalho de doutorado é de natureza aplicada, com objetivos de caráter descritivo/explicativo, utilizando procedimentos e estratégias de pesquisa experimental e estudos de caso.

Trata-se de uma pesquisa aplicada por utilizar o conhecimento adquirido nas áreas de Computação, Psicologia e Educação para o desenvolvimento de mecanismos mais efetivos para apoiar a formação de grupos em ambientes CSCL. Os objetivos são de caráter descritivo dado que este trabalho realiza uma análise minuciosa sobre a influência dos traços de personalidade na formação de grupos baseados em teorias de aprendizagem colaborativa; e explicativo por buscar compreender as causas e efeitos dessa influência. Quanto aos procedimentos, o método utilizado baseia-se no desenvolvimento de pesquisa experimental e estudos de caso, pois pretende-se manipular aspectos da realidade em busca de novos mecanismo (modelo formal e algoritmo computacional) para relacionar os traços de personalidade à teorias de aprendizagem e automatizar a formação de grupos em ambientes CSCL. Vale destacar que todos os estudos (ex.: estudo experimental, estudo de caso) realizados durante o desenvolvimento deste trabalho avaliaram o resultado da colaboração com respeito à aprendizagem e analisando sua relação com os traços de personalidade.

\section{Etapas de Pesquisa e Resultados}

Para alcançar o objetivo definido para este trabalho, foram elaboradas três questões de pesquisa cujas soluções são detalhadas nas próximas subseções.

\subsection{QP01 - Qual a relação entre os Traços de Personalidade e o Aprendizado, Satisfação e Motivação dos Grupos de Aprendizagem baseados em Teorias de Aprendizagem Colaborativa?}

\subsubsection{Descrição}

Para responder à QP01 foi elaborado um estudo experimental para investigar a influência dos traços de personalidade nas variáveis de aprendizagem, satisfação e motivação dos grupos apoiados pela teoria de aprendizagem Peer Tutoring [Endlsey 
1980] que permite aos alunos desempenharem os papéis de Tutor e Tutelado. Foram elaborados três grupos distintos de hipóteses, um para cada variável. A hipótese alternativa $\left(\mathrm{H}_{1}\right)$ supõe que os traços de personalidade ${ }^{2}$ rigidez mental, extroversão e emocionalidade possuem influência no aprendizado, motivação e satisfação dos grupos participantes de uma sessão colaborativa apoiada pela teoria Peer Tutoring [Endlsey 1980]. Por outro lado, a hipótese nula $\left(\mathrm{H}_{0}\right)$ supõe que não possui influência.

Esse estudo foi realizado com 156 alunos do $7^{\circ}, 8^{\circ}$ e $9^{\circ}$ anos do ensino fundamental e foi executado em três encontros com um intervalo de aproximadamente 15 dias. No primeiro encontro (Fase A), os participantes responderam individualmente ao pré-teste de conhecimento e teste de personalidade. $O$ pré-teste mediu $o$ conhecimento atual dos alunos sobre o conteúdo a ser abordado nas atividades em grupo e o teste de personalidade EPQ-J [Eysenck, 2013] avaliou os traços dos alunos nas dimensões: emocionalidade, extroversão e rigidez mental.

No segundo encontro (Fase B), os alunos foram agrupados em duplas para a realização das atividades em grupo. Além disso, os alunos responderam individualmente três testes: (1) satisfação em trabalhar com o colega antes e depois da intervenção, utilizando o teste SAM [Lang, 1980]; (2) motivação sobre o desenvolvimento das atividades em grupo com base nas subescalas de interesse/aproveitamento, percepção de competência, percepção de escolha e pressão/tensão utilizando o questionário IMI [Deci e Ryan1985] e (3) pós-teste para avaliar o conhecimento imediatamente adquirido após a conclusão das atividades em grupo.

Por fim, no terceiro encontro (Fase C) foi aplicado o teste de retenção que avaliou o conhecimento fixado (retido) pelos alunos após o período de 15 dias.

\subsubsection{Resultados}

As medidas usadas para caracterizar os grupos em termos dos traços de personalidade foram: elevação e variabilidade [Peeters et al. 2006]. A elevação foi calculada pela "média dos escores" do traço de personalidade dos membros do grupo e a variabilidade foi computada pelo "escore do desvio padrão" para determinado traço de personalidade. Para análise dos dados foi utilizado o coeficiente de correlação de Pearson para calcular a correlação entre a elevação e variabilidade dos traços de personalidade e as variáveis de aprendizado, motivação e satisfação ${ }^{3}$. As correlações significativas, $p<0,05$, foram assinaladas com um asterisco.

Com respeito ao aprendizado, a hipótese nula $\left(\mathrm{H}_{0}\right)$ não foi aceita para os traços Rigidez Mental (RM) e Emocionalidade (EM). Conforme mostrado pela Tabela 1, os resultados mostraram que esses traços tiveram influência significativa na média de aprendizagem do grupo. A variável "elevação do traço RM" apresentou correlação negativa e significativa com a variável "média de acertos na Fase $C$ " ( $r=-, 296 ; p=$ ,008) e "média do aprendizado retido" $(\mathrm{r}=-, 278 ; \mathrm{p}=, 014)$. Isso indica que quanto menor a elevação do traço RM no grupo, maior a média de acertos do grupo no teste de retenção (Fase C) e maior o aprendizado retido (Fase C - Fase A). Vale ressaltar que

\footnotetext{
2 Seguindo a recomendação de Eysenck (2013), neste estudo os termos "psicoticismo" e "neuroticismo" foram substituídos, respectivamente, pelos termos "rigidez mental" e "emocionalidade".

3 Para a variável satisfação a hipótese nula foi aceita $\left(\mathrm{H}_{0}\right)$, ou seja, não foi encontrada correlação significativa com nenhum dos traços.
} 
VIII Congresso Brasileiro de Informática na Educação (CBIE 2019)

Anais dos Workshops do VIII Congresso Brasileiro de Informática na Educação (WCBIE 2019)

essa relação foi significativa entre os alunos que desempenharam o papel de Tutelado. A variável "variabilidade do traço EM" também apresentou correlação negativa e significativa com a medida de aprendizado "média de acertos na Fase C" $(r=-, 233 ; p=$ ,040). Nesse caso, observa-se que grupos homogêneos (em termos de EM) tiveram melhor média de aprendizagem no teste de retenção (Fase C).

Tabela 1. Correlação entre a elevação do traço RM e variabilidade do traço EM e as variáveis de aprendizagem.

\begin{tabular}{|l|l|l|l|l|l|l|}
\hline & & $\begin{array}{l}\text { Média de } \\
\text { acertos na } \\
\text { Fase A }\end{array}$ & $\begin{array}{l}\text { Média de } \\
\text { acertos na } \\
\text { Fase B }\end{array}$ & $\begin{array}{l}\text { Média de } \\
\text { acertos na } \\
\text { Fase C }\end{array}$ & $\begin{array}{l}\text { Média do } \\
\text { aprendizado } \\
\text { imediato (B-A) }\end{array}$ & $\begin{array}{l}\text { Média do } \\
\text { aprendizado } \\
\text { retido (C-A) }\end{array}$ \\
\hline $\begin{array}{l}\text { Elevação do } \\
\text { traço RM }\end{array}$ & r &,- 104 &,- 072 &,$- 296^{*}$ &,- 046 &,$- 278^{*}$ \\
\cline { 2 - 7 } $\begin{array}{l}\text { Variabilidade } \\
\text { do traço EM }\end{array}$ & r &,- 1264 &, 532 &, 008 &, 691 &, 014 \\
\cline { 2 - 7 } & Sig. &, 287 &,- 087 &,$- 233^{*}$ &,- 056 &,- 209 \\
\hline
\end{tabular}

Com relação à motivação, a hipótese nula $\left(\mathrm{H}_{0}\right)$ não foi aceita para os traços Extroversão (EX) e Emocionalidade (EM). Conforme mostrado pela Tabela 2, a variável "variabilidade do traço EX" apresentou correlação positiva e significativa ( $\mathrm{r}=$ ,246; $p=030)$ com a variável "média de pressão e tensão". Isso indica que quanto mais heterogêneos os grupos (em termos de EX), mais os alunos se sentiram tensos, pressionados e/ou nervosos em realizar as atividades em grupo. A variável "elevação do traço EM", por outro lado, apresentou correlação negativa e significativa com a "variabilidade do traço EM" ( $\mathrm{r}=-, 227 ; \mathrm{p}=, 046)$ e "média do interesse e aproveitamento" ( $\mathrm{r}=-, 248 ; \mathrm{p}=, 029)$. Isso indica que quanto menor a elevação do traço EM do grupo (membros com estabilidade emocional), maior a média de interesse e aproveitamento em relação às atividades desenvolvidas.

Com base nos papéis foi encontrada correlação positiva e significativa $(\mathrm{r}=$ ,229; p = ,044) entre o traço EM e a variável de motivação "pressão e tensão" para os alunos que desempenharam o papel de Tutor. Nesse caso, quanto maior o nível do traço EM do aluno Tutor, maior a pressão e tensão na realização das atividades.

Tabela 2. Correlação entre a variabilidade do traço EX e elevação do traço EM e as variáveis de motivação.

\begin{tabular}{|c|c|c|c|c|c|c|c|}
\hline & & $\begin{array}{l}\text { Variabi- } \\
\text { lidade do } \\
\text { traço EM }\end{array}$ & $\begin{array}{l}\text { Média de } \\
\text { interesse e } \\
\text { aproveitamento }\end{array}$ & $\begin{array}{l}\text { Média de } \\
\text { percepção de } \\
\text { competência }\end{array}$ & $\begin{array}{l}\text { Média de } \\
\text { percepção } \\
\text { de escolha }\end{array}$ & $\begin{array}{l}\text { Média } \\
\text { de } \\
\text { pressão } \\
\text { e tensão }\end{array}$ & $\begin{array}{l}\text { Média da } \\
\text { motivação } \\
\text { intrínseca } \\
\text { geral }\end{array}$ \\
\hline \multirow{2}{*}{$\begin{array}{l}\text { Variabilidade } \\
\text { do traço EX }\end{array}$} & $r$ & \multirow{2}{*}{$\mathrm{N} / \mathrm{A}^{4}$} &,- 038 &,- 031 &,- 031 & ,246* &,- 153 \\
\hline & Sig. & &, 744 & ,789 & ,787 &, 030 & , 180 \\
\hline \multirow{2}{*}{$\begin{array}{l}\text { Elevação do } \\
\text { traço EM }\end{array}$} & $r$ &,$- 277 *$ &,$- 248^{*}$ &, 045 &,- 111 &,- 138 &,- 156 \\
\hline & Sig. &, 046 &, 029 & 698 &, 334 & ,229 & 173 \\
\hline
\end{tabular}

\subsection{QP02 - Como criar um Modelo Formal que relacione os Traços de} Personalidade às Teorias de Aprendizagem Colaborativa?

\subsubsection{Descrição}

Para responder a QP02 foi desenvolvido um Modelo Formal, baseado em ontologias

\footnotetext{
${ }^{4}$ N/A significa que a relação entre a variabilidade do traço EX e EM não se aplica.
} 
VIII Congresso Brasileiro de Informática na Educação (CBIE 2019)

Anais dos Workshops do VIII Congresso Brasileiro de Informática na Educação (WCBIE 2019)

[Borst 1997], para representar novos papéis de aprendizagem definidos a partir da combinação dos traços de personalidade e teorias de aprendizagem colaborativa.

No contexto colaborativo, Isotani et al. (2009) desenvolveram a "ontologia de papéis" como uma estrutura ontológica para representação dos papéis de aprendizagem. Essa ontologia é formada pelas condições necessárias (obrigatórias) e desejadas (opcionais) para que um aluno possa desempenhar o papel e pelo comportamento esperado pelo aluno no grupo. A decisão por utilizar essa ontologia foi embasada em estudos científicos [Andrei et al. 2015] e nos resultados do estudo experimental apresentado na Seção 3.1.2. A partir desses estudos foi observado que os traços de personalidade influenciam a forma como os alunos interagem no grupo e que essa forma de interação pode estar relacionada ao papel desempenhado no grupo.

\subsubsection{Resultados}

Para relacionar os traços de personalidade às teorias de aprendizagem colaborativa foi criado um procedimento formado pela seguinte sequência de passos:

(1) Selecionar um conjunto de traços de personalidade;

(2) Identificar as características do traço de personalidade;

(3) Identificar os papéis que os alunos podem desempenhar no grupo com base nas teorias de aprendizagem colaborativa;

(4) Obter as características (condições necessárias/desejadas) dos papéis;

(5) Relacionar as características dos traços e as características dos papéis para a criação dos Papéis Colaborativos Afetivos (PCAs).

A Figura 1 apresenta um exemplo do Modelo Formal, criado com base no procedimento anterior, em que os PCAs (à direita) são especializações dos papéis colaborativos apresentados à esquerda.

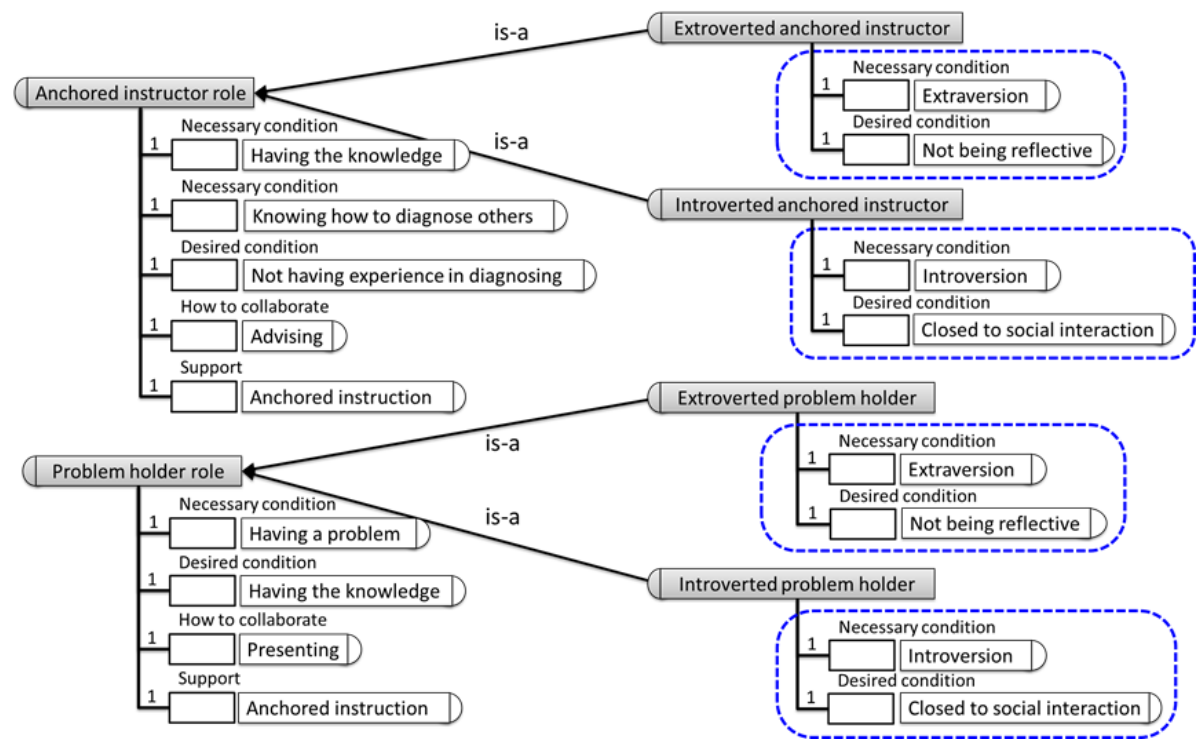

Figura 1. Ontologia de papéis colaborativos afetivos. À esquerda estão os papéis de instrutor e instruído e à direita os quatro novos PCAs.

Os PCAs ilustrado na Figura 1 foram criados a partir da combinação dos traços de personalidade extroversão (extroverted)/introversão (introverted) e os papéis de instrutor (anchored instructor)/instruído (problem holder) pertencentes a teoria de 
VIII Congresso Brasileiro de Informática na Educação (CBIE 2019)

Anais dos Workshops do VIII Congresso Brasileiro de Informática na Educação (WCBIE 2019)

aprendizagem Anchored Instruction [CTGV 1992]. Esse modelo em particular foi avaliado por meio de um estudo de caso desenvolvido por Reis et al. (2016). Outro modelo criado seguindo o mesmo procedimento poder ser visto em Reis et al. (2017).

\subsection{QP03 - Como criar um algoritmo computacional, que apoie o processo de formação de grupos de aprendizagem, usando os traços de personalidade combinados às teorias de aprendizagem colaborativa?}

\subsubsection{Descrição}

Para responder a questão de pesquisa QP03, está seção apresenta um algoritmo de formação de grupos desenvolvido na linguagem PHP, denominado Group Formation USIng ONtology and Personality Trait (G-FusionPT), que utiliza os novos papéis de aprendizagem (PCAs) gerados a partir do procedimento apresentado na Seção 3.2.2. O algoritmo é formado por duas etapas principais: (1) determinar quais teorias de aprendizagem atendem aos alunos e (2) formar os grupos de aprendizagem.

A primeira etapa consiste em verificar se o aluno possui os pré-requisitos para desempenhar o papel de uma teoria de aprendizagem colaborativa. Caso positivo, a teoria atende ao aluno e o mesmo é inserido em uma lista de candidatos composta pelo nome da teoria, objetivo de aprendizagem, papel a ser desempenhado e estratégia de aprendizagem. Essa lista em conjunto com o tamanho do grupo servirá de entrada para o algoritmo de formação de grupos mostrado no formato de pseudocódigo na Tabela 3.

\section{Tabela 3. Pseudocódigo para formar grupos de aprendizagem.}

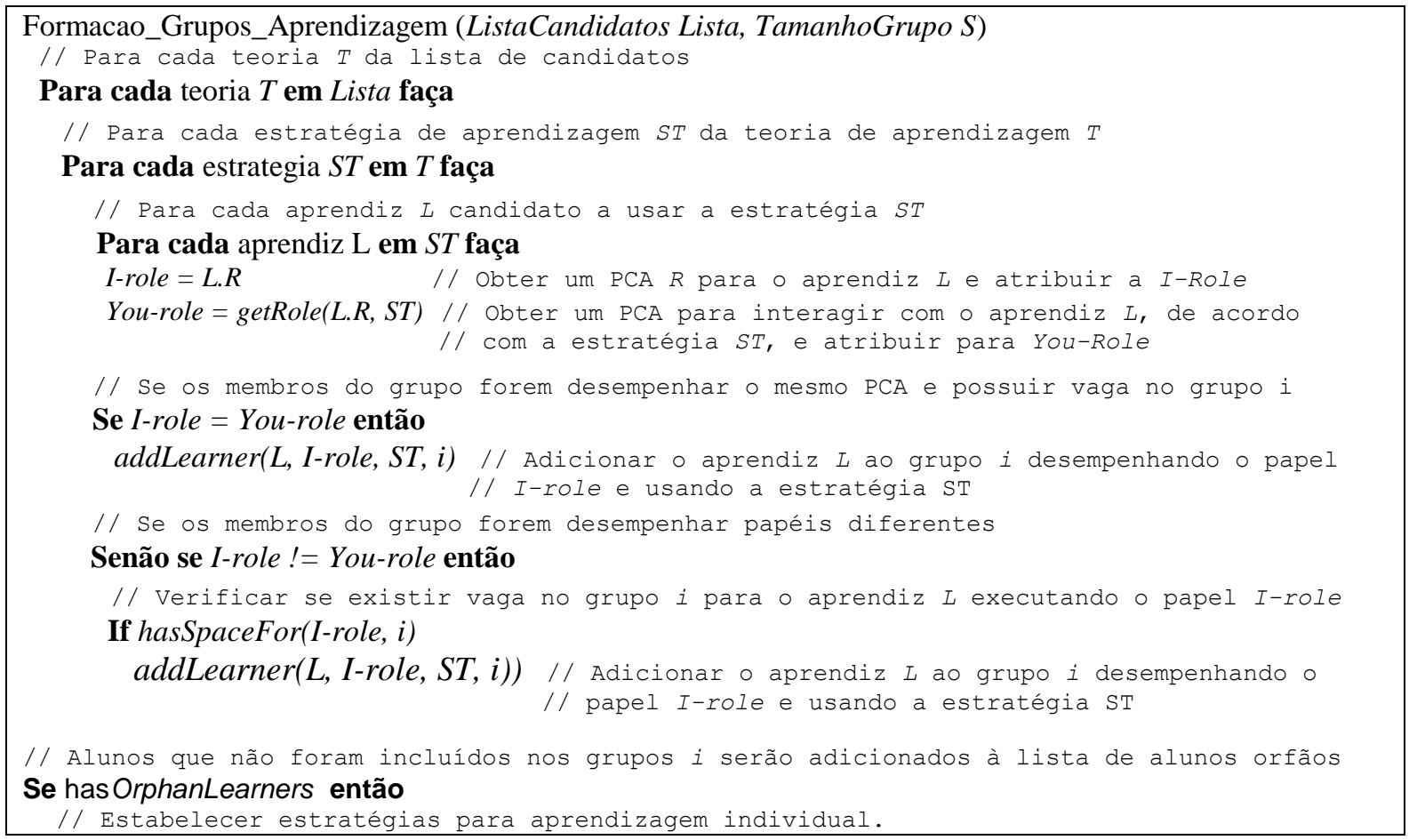

\subsubsection{Resultados}

Para execução do algoritmo G-FusionPT foi criado o protótipo de um sistema computacional, denominado Visual Tool for Effective Group Formation (VISO-EGO), que permite comparar os resultados do algoritmo proposto com dois outros algoritmos 
VIII Congresso Brasileiro de Informática na Educação (CBIE 2019)

Anais dos Workshops do VIII Congresso Brasileiro de Informática na Educação (WCBIE 2019)

de formação de grupos: G-Fusion e Randômico. O algoritmo G-Fusion baseia-se especificamente nas teorias de aprendizagem colaborativa [Isotani et al. 2009] e o Randômico não se baseia em qualquer critério ou estratégia para formar os grupos.

No intuito de avaliar a efetividade do algoritmo G-FusionPT em comparação com os algoritmos G-Fusion e Randômico foi gerada uma amostra simulada de 300 alunos utilizando o método de simulação Monte Carlo [Harrison 2010]. A Figura 2 mostra os resultados dos algoritmos, Randômico, G-Fusion e G-FusionPT, após 30 execuções a partir do sistema VISO-EGO considerando grupos com três membros.

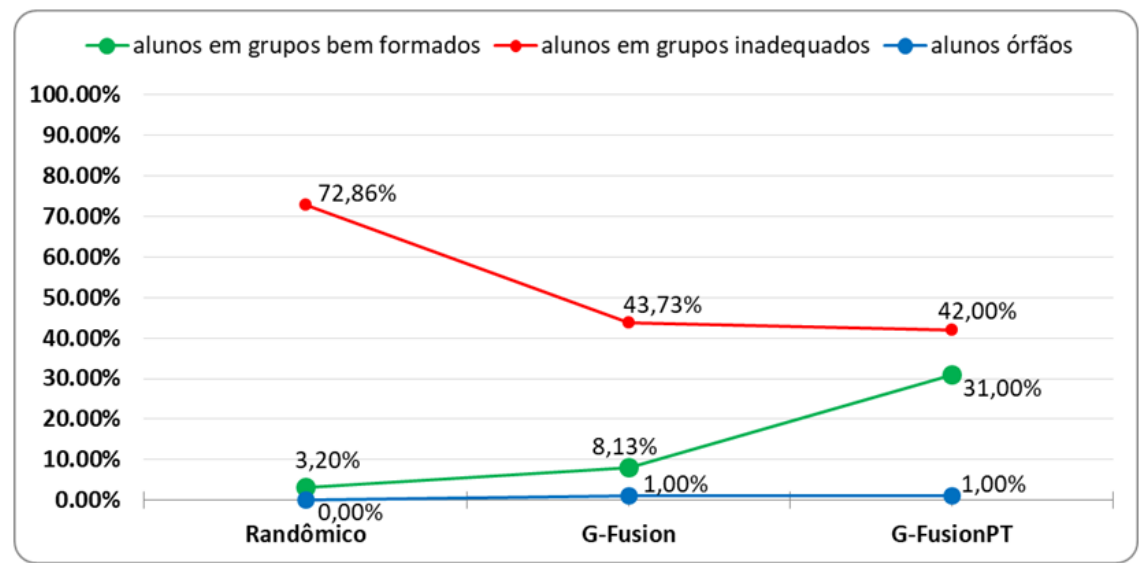

Figura 2. Percentual médio dos alunos em "grupos bem formados", "grupos inadequados" e "órfãos" para cada algoritmo e grupos com três membros.

Para a avaliação dos algoritmos foram utilizadas três medidas: (1) percentual médio de alunos em grupos bem formados (grupos com 100\% de validade), (2) percentual médio de alunos em grupos inadequados (grupos com $0 \%$ de validade), (3) percentual médio de alunos órfãos. A partir Figura 2 observa-se que o algoritmo GFusionPT foi o que obteve menor percentual médio de "alunos em grupos inadequados" e maior percentual de "alunos em grupos bem formados". Com respeito aos alunos órfãos os três algoritmos tiveram percentuais bem próximos.

\section{Conclusões}

Este trabalho foi guiado por três questões de pesquisa para apresentar os resultados de uma pesquisa de doutorado. Os resultados para a QP01 no quesito aprendizagem mostraram que grupos formados por membros com baixo escore no traço rigidez mental tiveram melhor aprendizado comparado aos grupos formados por membros com alto escore. Apesar desse resultado possuir relação com trabalhos na literatura [Heaven et al. 2007], acredita-se que o uso de teorias de aprendizagem colaborativa que explorem a criatividade dos alunos com alto escore em rigidez mental possa alterar essa relação, dado que a criatividade é uma característica desse traço. Sobre o traço emocionalidade, os resultados mostraram que quanto menor a diversidade desse traço entre os membros, maior o aprendizado do grupo. Apesar de alguns estudos não recomendarem membros com alto escore em emocionalidade nos grupos de aprendizagem [Molleman et al. 2004], nenhuma relação significativa foi encontrada pelo estudo experimental realizado.

No quesito motivação, constatou-se que quanto maior a diversidade do traço extroversão entre os membros, maior a "pressão e tensão" do grupo na realização das 
atividades. Embora valores elevados para "pressão e tensão" tendem a ser vistos como negativos, nada se pode afirmar sobre o seu efeito no grupo. De acordo com D'Mello et al. (2014), alguns estados afetivos tratados como negativos (ex.: confusão), podem ser positivos para aprendizagem quando apropriadamente induzidos e regulados. Sobre o traço emocionalidade, os resultados mostraram que grupos formados por membros com baixo escore apresentaram maior "interesse e aproveitamento" nas atividades em grupo. Esse resultado é apoiado por trabalhos na literatura como Vianen e De Dreu (2001).

Com respeito a QP02, os principais benefícios do Modelo Formal são: (1) permitir a criação de novos PCAs a partir da combinação entre qualquer conjunto de traços de personalidade e qualquer conjunto de teorias de aprendizagem colaborativa; (2) possibilitar a reutilização dos PCAs em outras situações de aprendizagem dado que sua representação é baseada em ontologias; (3) ser aplicável em contextos reais de aprendizagem [Reis et al. 2016, 2017]. Vale salientar ainda que o tipo de relação do Modelo Formal proposto neste trabalho não foi encontrado na literatura.

Sobre a QP03, o algoritmo G-FusionPT obteve melhor resultado com relação ao número de alunos em grupos bem formados ( $100 \%$ válidos) e inadequados ( $0 \%$ válidos) quando comparado aos algoritmos Randômico e G-Fusion. Apesar da existência de diversos algoritmos computacionais para apoiar a formação de grupos em ambientes CSCL [Borges et al. 2018], não foram encontrados na literatura estudos fundamentados em teorias ou estratégias pedagógicas e que considerassem os traços de personalidade no agrupamento dos alunos [Reis et al. 2015]. Logo, o algoritmo G-FusionPT pode ser visto como um dos pioneiros a modelar os novos papéis de aprendizagem (PCAs) computacionalmente.

\section{Referências}

Andrei, F., Mancini, G., Mazzoni, E., Russo, P., Baldaro, B. (2015). Social status and its link with personality dimensions, trait emotional intelligence, and scholastic achievement in children and early adolescents. Learning and Individual Differences, Netherlands: Elsevier Science, v. 42, p. 97-105.

Borges, S., Mizoguchi, R.; Bittencourt, I. I., Isotani, S. (2018). "Group formation in cscl: A review of the state of the art". In: Cristea, A. I., Bittencourt, I. I., Lima, F. (Ed.). Communications in Computer and Information Science. Springer, v. 832, p. 71-88.

Borst, W. (1997). Construction of Engineering Ontologies for Knowledge Sharing and Reuse. Tese (Phd thesis) - University of Twente, Netherlands.

CTGV - Cognition and Technology Group at Vanderbilt) (1992). "Anchored instruction in science education". In: Duschl, R., Hamilton, R. (Ed.). Philosophy of science, cognitive psychology, and educational theory and practice. SUNY Press, p. 244-273.

Deci, E. L., Ryan, R. M. (1985). Intrinsic motivation and self-determination in human behavior, Springer US. 372 p.

D’Mello, S.; Lehmann, B.; Pekrun, R.; Graesser, A. (2014). Confusion can be beneficial for learning. Learning and Instruction, v. 29, p. 153-170.

Endlsey,W. R. (1980). Peer Tutorial Instruction, Educational Technology Publications. (Instructional design library).

Eysenck, H. J. (2013). Questionário de Personalidade para Crianças e Adolescentes (EPQ$\mathrm{J})$ : livro de instruções para uso profissional no Brasil. Vetor. 
VIII Congresso Brasileiro de Informática na Educação (CBIE 2019)

Anais dos Workshops do VIII Congresso Brasileiro de Informática na Educação (WCBIE 2019)

Farhangian, M., Purvis, M., Purvis, M., BASTIN, T. R. S. (2015). "The effects of temperament and team formation mechanism on collaborative learning of knowledge and skill in shortterm projects". In: Koch, F.; Guttmann, C.; Busquets, D. (Ed.). Advances in Social Computing and Multiagent Systems. Cham: Springer International Publishing, p. 48-65.

Heaven, P. C., Ciarrochi, J., Vialle, W. (2007). Conscientiousness and eysenckian psychoticism as predictors of school grades: A one-year longitudinal study. Personality and Individual Differences, v. 42, n. 3, p. 535-546.

Harrison, R. L. (2010). "Introduction to monte carlo simulation". AIP Conference Proceedings, v. 1204, p. 17-21.

Isotani, S., Mizoguchi, R. (2008). "Adventures in the boundary between domainindependent ontologies and domain content for cscl”. In: Lovrek, I.; Howlett, R. J.; Jain, L. C. (Ed.). Knowledge-Based Intelligent Information and Engineering Systems. Berlin, Heidelberg: Springer Berlin Heidelberg, p. 523-532.

Jung, C. F. (2004). Metodologia para pesquisa \& desenvolvimento: aplicada a novas tecnologias, produtos e processos, Axcel Books do Brasil Ltda.

Isotani, S., Inaba, A.; Ikeda, M., Mizoguchi, R. (2009). An ontology engineering approach to the realization of theory-driven group formation. International Journal of ComputerSupported Collaborative Learning, v. 4, n. 4, p. 445-478.

Lang, P. (1980) Self-assessment manikin. Gainesville, FL: The Center for Research in Psychophysiology, University of Florida.

Molleman, E., Nauta, A., Jehn, K. A. (2004) Person-job fit applied to teamwork: A multilevel approach. Small Group Research, v. 35, n. 5, p. 515-539.

Nunes, M. A. S. N., Bezerra, J. S., Reinert, D., Moraes, D., Silva, É. P., Pereira, A. J. S. (2010). "Computação Afetiva e sua influência na personalização de Ambientes Educacionais: gerando equipes compatíveis para uso em AVAs na EaD”. In: Machado, G. J. C. (Ed.).Educação e Ciberespaço: Estudos, propostas e desafios. Aracaju: Virtus Editora, p. 308-347.

Peeters, M. A.; Tuijl, H. F. van; Rutte, C. G.; Reymen, I. M. M. J. (2006). Personality and team performance: a meta-analysis. European journal of personality, John Wiley and Sons Ltd, 20(5), p. 377-396.

Reis, R. C., Rodriguez, C. L., Challco, G. C., Lyra, K. T., Marques, L. B., Jaques, P. A., Bittencourt, I. I., Isotani, S. (2016). Step towards a model to bridge the gap between personality traits and collaborative learning roles. $I x D \& A$, n. 28, p. 124-144.

Reis, R. C. D., Rodriguez, C. L., Lyra, K. T., Jaques, P. A., Bittencourt, I. I.,Isotani, S. (2015). Estado da Arte sobre Afetividade na Formação de Grupos em Ambientes Colaborativos de Aprendizagem. RBIE, 23(3), p. 113-130.

Reis, R. C. D., Rodriguez, C. L., Lyra, K. T., Isotani, S. (2017). Estrutura Ontológica para representar Papéis Colaborativos Afetivos em ambientes CSCL. RBIE, 25(2), p. 87-106.

Spoelstra, H., Rosmalen, P. van, Sloep, P. (2014). Toward project-based learning and team formation in open learning environments. Journal of Universal Computer Science, 20 (1), p. 57-76.

Vianen, A. E. van; De Dreu, C. K. (2001). Personality in teams: Its relationship to social cohesion, task cohesion, and team performance. European Journal of Work and Organizational Psychology, 10(2), p. 97-120. 